\title{
New methodology to explore the role of visualisation in aircraft design tasks
}

\section{Evelina Dineva*, Arne Bachmann, Erwin Moerland, Björn Nagel and Volker Gollnick}

\author{
Deutsches Zentrum für Luft- und Raumfahrt e.V. (DLR), \\ German Aerospace Center, \\ Blohmstr. 18, 21079 Hamburg, Germany \\ E-mail: Evelina.Dineva@dlr.de \\ E-mail: Arne.Bachmann@dlr.de \\ E-mail: Erwin.Moerland@dlr.de \\ E-mail: Bjoern.Nagel@dlr.de \\ E-mail: Volker.Gollnick@dlr.de \\ *Corresponding author
}

\begin{abstract}
A toolbox for the assessment of engineering performance in a realistic aircraft design task is presented. Participants solve a multidisciplinary optimisation (MDO) task by means of a graphical user interface (GUI). The quantity and quality of visualisation may be systematically varied across experimental conditions. The GUI also allows tracking behavioural responses like cursor trajectories and clicks. Behavioural data together with evaluation of the generated aircraft design can help uncover details about the underlying decision making process. The design and the evaluation of the experimental toolbox are presented. Pilot studies with two novice and two advanced participants confirm and help improve the GUI functionality. The selection of the aircraft design task is based on a numerical analysis that helped to identify a 'sweet spot' where the task is not too easy nor too difficult.
\end{abstract}

Keywords: empirical performance evaluation; visualisation; aircraft design; multidisciplinary optimisation; MDO; multidisciplinary collaboration; collaborative engineering.

Reference to this paper should be made as follows: Dineva, E., Bachmann, A., Moerland, E., Nagel, B. and Gollnick, V. (2014) 'New methodology to explore the role of visualisation in aircraft design tasks', Int. J. Agile Systems and Management, Vol. 7, Nos. 3/4, pp.220-241

Biographical notes: Evelina Dineva is a Research Scientist at the Institute for Air Transportation Systems, German Aerospace Center (DLR). She seeks to uncover mechanisms of collaborative engineering in order to help improve working and learning environments. Her approach is based on empirical research and formal mathematical methods. She received her Mathematics Diploma from the University of Hamburg and $\mathrm{PhD}$ in Neuroscience from the International Graduate School of Neuroscience, Ruhr-University Bochum. She gained post-doctoral experience in cognitive psychology at Indiana University, Bloomington, IN and at the University of Iowa, Iowa City, IA where she is an affiliate member of the Delta Center. 


\begin{abstract}
Arne Bachmann received his Magister Artium degree in Phonetics and Communication Research from the University of Bonn in 2007, specialising in speech technology. He has worked for several years in software development of scientific service-oriented architectures and has taken new responsibilities in project management of regional and international research projects since 2011 . $\mathrm{He}$ is currently engaged in hardware and software integration for the integrated design laboratory to enable engineers find better ways in distributed as well as co-located collaboration.
\end{abstract}

Erwin Moerland received his Master degree from the Faculty of Aerospace Engineering at Delft University of Technology in 2011, focusing on overall aircraft design with aerodynamics and aircraft structures and mechanics as major sub-themes. During his student years, he has been involved in the set-up of the bachelor courses on mechanics and aircraft structural analysis and was a board member of the society of aerospace engineering students. Currently, he is a $\mathrm{PhD}$ student in the Integrated Aircraft Design group within the Institute for Air Transportation Systems of the German Aerospace Center (DLR). His fields of research include interdisciplinary communication and collaborative design within multidisciplinary aircraft design and optimisation (MDO).

Björn Nagel is Deputy Head of the DLR Institute of Air Transportation Systems; Head of the DLR Department Integrated Aircraft Design; DLR Programmatic Spokesman for the research topic Concepts \& Integration; Coordinator of the Technical Committee on Aircraft Design in the Council of European Aerospace Societies (CEAS); Lecturer for Aircraft Design at the Hamburg University of Technology, TUHH; and leader of various multidisciplinary, trans-organisational and trans-national research projects. BN's research activities are focused on aircraft design, multidisciplinary design optimisation (MDO), participative MDO, and human collaboration processes.

Volker Gollnick holds the chair of the Institute for Air Transportation Systems of the German Aerospace Center (DLR) at the Technical University Hamburg-Harburg. He is particularly interested in operational improvements and changes of sustainable but efficient air transportation systems. Integration and collaboration of the various stakeholders is his special focus, when he is researching on new aircraft, airports and aircraft operations. He started his professional career at the German Forces Flight Test Center, and has held senior positions at Eurocopter and EADS especially working on pilot handling qualities and cockpit systems MMI. He received his Diploma in Mechanical and Aerospace Engineering from the Technical University of Braunschweig and his PhD from the Technical University of Munich.

This paper is a revised and expanded version of a paper entitled 'Empirical performance evaluation in collaborative aircraft design tasks' presented at 20th International Conference on Concurrent Engineering, Melbourne, Australia, 2-5 September 2013.

\title{
1 Introduction
}

Given the sheer complexity of many current day engineering projects, it has become impossible for individuals to comprehend the entire system, and collaboration among experts from diverse disciplines has become highly relevant for the success of research and design departments (Kolonay, 2013). Consider, for instance, the complexity of 
aircraft design (Felder and Collopy, 2012), and the multitude of external and internal players involved in it. On one hand, close cooperation between designers and stakeholders is necessary in order to specify goals about future aircraft concepts. On the other hand, specific design projects require that experts from diverse science, technology, engineering, and mathematics (STEM) fields collaborate on a high technical level. Researchers at the Institute for Air Transportation Systems at the German Aerospace Center (DLR) work to bridge both these cooperation levels. Externally, they interact closely with stakeholders like airports, airlines, aircraft and air traffic management to develop holistic understanding of air-transportation. Internally, this knowledge provides significant boundary conditions for aircraft design projects and flows into the design of interdisciplinary collaborative tools (Böhnke et al., 2011; Nagel et al., 2012, 2013).

The day-to-day practise of high-level integrative work exposed the need to gain explicit knowledge about the mechanisms of collaborative engineering. This realisation is shared with Ilan Kroo and Juan Alonso at Stanford University, who proposed a third generation multidisciplinary aircraft design view, highlighting the need for understanding decision processes in multidisciplinary teams. We consider the improvement of collaboration among engineers from different sub-disciplines and between engineers and stakeholders as a major challenge toward improving engineering education and toward providing conditions for better engineering practise and success. As a mean to that end, the goal of this paper is to address the question of how engineering performance can be assessed depending on a given working context - the context is what can be manipulated to encourage performance. Understanding the relationship of performance and context is clearly an empirical question that needs to be addressed with methods from the psychological and sociological sciences. Such methods are adapted to develop an experimental toolbox. The toolbox incorporates a graphical user interface (GUI) that allows one to evaluate how different modes of visualisation influence the performance of engineers when they solve an aircraft design task - formulated as a multidisciplinary optimisation (MDO) problem. With this study a base-line experiment is created, which currently is the basis of a controlled experimental study. Note that, at the current stage, only individual performance can be accessed. However, it is intended to extend the toolbox to study collaborative engineering.

\section{Related work}

Support of multidisciplinary working projects range from visualisation techniques (Eppler and Lengler, 2007) and knowledge-based support tools (Elgh, 2013; Fruchter et al., 1996; la Rocca and van Tooren, 2008; Oellrich and Mantwill, 2013; Patel et al., 2012) to entire facilities (Bachmann et al., 2012; Dineva et al., 2013; Schubert et al., 2010; Xu et al., 2013). Noteworthy is a tool for visualisation of airline disruption data that was developed based on extensive interviews of airline operations controllers, (Rosenthal et al., 2013). Reversely, empirical research is also applied to specify ability requirements for professionals. Eye-tracking systems allow to evaluate the ability to recognise and attend to critical situations, which has recently been introduced into the selection process for air-traffic controllers and pilots (Hasse et al., 2012, 2013). Critical in this research is to find appropriate scanning behaviour when participants assess information - human behaviour has to be well adapted to the given visualisation tools (Eißfeldt, 2011; Eißfeldt et al., 2011). That perception, cognition, and action form a 
coupled dynamic system is also shown in basic cognitive science research (Breazeal et al., 2005; Buss and Spencer, 2012; Erlhagen and Schöner, 2002; Simmering et al., 2008; Spencer et al., 2009). Thus, changing the visual context and behavioural history have a profound impact on how people make decisions. The present study exploits the close relationship of action, perception and cognition to help uncover how the quality and quantity of visual information might influence engineers' performance during a design task. In the long run, this research aims to find requirements and guidelines for how to improve the Integrated Design Laboratory (IDL; see Bachmann et al., 2012).

The scope of this paper is on the methodology of developing the experimental toolbox, which is related to solving two major challenges. First is the selection of an appropriate MDO task, that is challenging but doable for the experimental target group, which is engineering students (presented in Section 3). Second is the provision of the experimental toolbox - a user interface, GUI - in which experimental conditions can be manipulated, and with which behavioural data can be collected (presented in Section 4). In Section 5, pilot studies with four subjects (two novice and two advanced engineering students) are presented, the results confirm that the feasibility of the MDO-GUI toolbox as a mean to study engineering performance. In addition, based on the pilot data analysis, critical improvements of the experimental toolbox are identified. In conclusion (Section 7), with the improvements in place, a complete experimental study can be conducted and extended.

\section{Prerequisites for experimental research}

The selection of an appropriate MDO design task is a central goal of this study and mandatory for the development of an experimental toolbox. Parts of this Section have been presented in Dineva et al. (2013).

\subsection{Software tools for experimentation}

To keep laboratory investigation close to real work scenarios, experimental studies are based upon VAMPzero (Bachmann et al., 2010; Böhnke et al., 2011), which is the current software tool used to study preliminary aircraft design configurations at DLR. VAMPzero calculates a mass-breakdown and global performance data by using a mix of statistical (handbook) and low-fidelity physical methods and models. Calculations are initialised with a dataset comparable to the Airbus aircraft A320-type that is provided as a CPACS file [CPACS is a xml-based common language for aircraft design (see Nagel et al., 2012)]. To create new designs, participants can interactively modify control parameters of the A320-type dataset and then iterate VAMPzero through a GUI (programmed in Matlab ${ }^{\circledR}$ ).

\subsection{Restriction of parameter settings}

The selection of adequate control and output parameters is mandatory for a good aircraft design task for engineering graduates to be able to perform. In several discussion sessions, our team (of engineers and cognitive scientists) deliberately settled for a relatively small number of control and output parameters. As control parameters, the aircraft's design range, wing span, and the engine's bypass ratio (BPR) were considered. 
A systematic numeric test was setup to investigate in detail their effects on the output parameters fuel mass, take-off mass (TOM), operating empty mass (OEM), and direct operating costs (DOC). In the subsequent analysis, we looked for global dependencies of the control on the output parameters to further narrow the experimental task. From the perspective of an operating airline, lower values are better for all of the above output parameters, therefore the optimisation goal of the task is to adjust the control parameters such that output parameters are minimised.

\subsubsection{Numeric testing procedure}

To evaluate if a given subset of control parameters indeed circumscribes a reasonable design task for engineering students, VAMPzero was repeatedly iterated. Combinations of three control parameters, the values of which were varied in small steps, were tested. Input settings and resulting output values were recorded for each iteration. Tests and the subsequent analysis were performed with Matlab ${ }$.

\subsubsection{Parameter analysis}

For analysis, the dependencies of output parameters on the input parameters were visualised. Most indicative were three-dimensional (3D) surface plots in which the local optima per design variable are highlighted. Examples are shown in Figures 1 and 2. Each subplot in Figure 1 shows how the DOC change for different wing span and bypass ratio settings and a fixed design range; the design range is varied between subplots and increases from the top left to the bottom right subplot. Figure 2 represents the same data, however rearranged such as to vary the engine's bypass ratio from low to high values across the subplots.

Figure 1 Parametric inter-dependencies: DOC levels (z-axes) depending on bypass ratio (x-axes), wing span (y-axes), and design range (varied over the subplots)
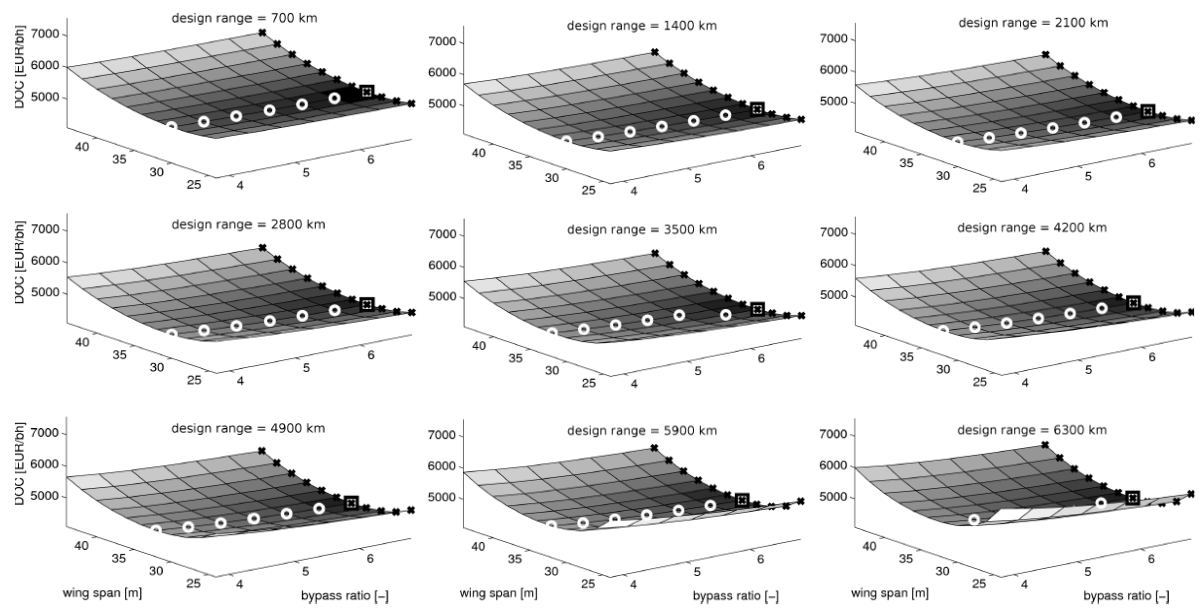

Notes: White circles indicate the DOC minima for each tested bypass ratio value, whereas black crosses indicate the DOC minima for each tested wing span value. The global minimum is highlighted using a large black square. Default A320-type setting is for design range $=3,500 \mathrm{~km}$ (middle subplot). 
Figure 2 Parametric inter-dependencies: DOC levels (z-axes) depending on design range (x-axes), wing span (y-axes), and bypass ratio (varied over the subplots)
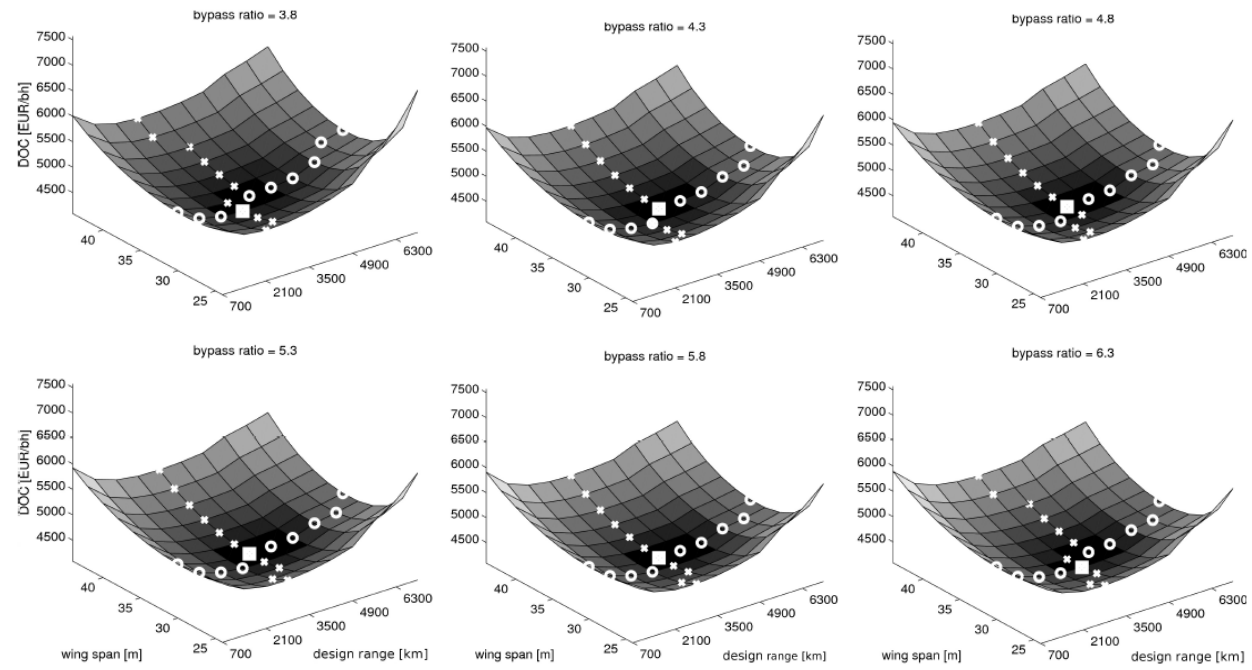

Notes: White circles indicate the DOC minima for each tested design range value, and white crosses indicate the DOC minima for each tested wing span value. The global minimum is highlighted using a filled white square. Default A320-type setting is for bypass ratio $=4.8$ (top right subplot).

Comparison of the subplots in Figure 1 shows a stronger curvature of the DOC surface for increasing design range. Therefore, the DOC shows a larger sensitivity to the remaining two parameters (wing span and design range) for increasing design range settings. The plots also show that overall costs are lower for designs that use engines with a high bypass ratio. For fixed design range and bypass ratio, a clear minimum considering the wing span dimension is observed. Adjusting the latter variable will be more difficult for the test persons, since the minimum is not located at the edge of the parameter range under consideration. Furthermore, the value of the absolute minimum to be attained will depend on the settings of the other values. The behaviour of the DOC minimum as function of wing span setting is caused by two conflicting optimisation criteria from aerodynamics and structural mechanics. Introducing a larger wing span will make the aircraft aerodynamically more efficient: a larger amount of air is deflected with a relatively lower velocity over the wing to generate the required lift force. Since the kinetic energy required to deflect the oncoming air is linearly dependent on the mass and quadratically dependent on the velocity, it is energetically more advantageous to have a large wing span. From a structural point of view, having a larger wing span is however disadvantageous: larger bending moments will occur due to increased moment vectors of the lift forces leading to higher material stress and the corresponding heavier structure needed. Conflicts that tap into knowledge from different sub-disciplines involved in aircraft design are necessary for the experimental task.

Different challenges concerning the design task are evident from Figure 2. For one, it shows that the choice of design range has a non-linear effect on the DOC. This might seem counter-intuitive as one might assume that, the shorter the design range, the cheaper 
both production and operation are. However, the direct operating costs are reported in Euros (EUR) per business hour (h) with a [EUR/h] unit. Shorter ranges mean reduced utilisation and more turnaround time on the ground, which is in fact a cost factor for the operating airline. As an engineering task, this is interesting since it introduces a component from a different discipline - economics - which in fact poses relevant constraints for engineering practise. The perspective of Figure 2 also exposes an interaction between wing span and design range, both of which have a non-linear influence on the DOC. The associated values in the subplots indicate that the aircraft design can be optimised globally by adjusting wing span and design range for the given engine at hand. All subplots show a similar shape and furthermore it is seen that the engine's bypass ratio does not affect the location of the global minimum largely (with respect to DOC). This can be explained due to the underlying calculation software, in which the relation between the bypass ratio of the engine and the corresponding effect on the engine mass is still to be incorporated. For the experiment, it is interesting to see if participants will exploit this independence - note that they will not have the global relationships among parameters available as displayed here (the task would be trivial otherwise).

After similar analyses of the effects of the control parameters on fuel mass, TOM, and OEM, the output parameter OEM was also selected for the experimental task. Traditionally, reducing mass is seen as critical within aircraft design. Optimising for a combination of both OEM and DOC is then particularly interesting, since counter-intuitive relations might occur. For example, for a set of aircraft requirements one could obtain a more DOC efficient aircraft, which is heavier than the configuration at the global mass minimum.

\subsubsection{Results: MDO task selection}

The main goal of the analysis is to narrow down a set of control and output parameters which specify a manageable but demanding task for engineering students. A summary of the results is given in Table 1 . In addition to specifying the control and output parameters, meaningful ranges and change-steps ( $\Delta$ s, i.e., pseudo-continuous or discrete) for the control parameters could be identified. We selected the ranges such that most of the designs are feasible, but allowed some extreme conditions in which VAMPzero yields a warning that the convergence in not possible - for instance, the selected wing span is too short to hold an engine with a given BPR. The feasibility ranges largely depend on the software used to calculate the aircraft properties according to the provided control parameters (see also Zill et al., 2012). Since VAMPzero is an empirical tool, the equations based on statistics limit the values of control parameters at hand. No change-steps are reported for the output parameters since these are actual outcomes of the calculations and not predefined like the controls. For the output parameters, the reported ranges serve, when applicable, for the purposes of plotting the results within their minimal possible range in the GUI. 
Table 1 Details for the control and output parameters, as selected for the experimental design task

\begin{tabular}{lccccc}
\hline Type & \multicolumn{3}{c}{ Control parameters } & \multicolumn{2}{c}{ Output parameters } \\
\hline Name & Design range & Wing span & Bypass ratio & DOC & OEM \\
Range & $350-7,000$ & $14-44$ & $3.5-7$ & $4,000-12,000$ & $3-130$ \\
$\Delta$ & Eight discrete & Continuous & Continuous & n/a & $\mathrm{n} / \mathrm{a}$ \\
Unit & {$[\mathrm{km}]$} & {$[\mathrm{m}]$} & {$[-]$} & {$[\mathrm{EUR} / \mathrm{h}]$} & {$[\mathrm{t}]$} \\
\hline
\end{tabular}

Notes: The symbol $\Delta$ shows in what change-steps the control parameters could be set in the GUI. Units for design range are [m] and for OEM are [kg].

\section{Experimental design}

The requirements on the experimental design are twofold. The first relates to the aircraft design task - it needs to be demanding but manageable for engineering undergraduates. Constraining such a task was done in Section 3.2. The second requirement relates to the experimental design - which and how much information should be displayed, or who should be tested in the different experimental condition. In sum, for the experiment we take into account:

a control and output parameters of the aircraft design

b quality and quantity of information about participants' design solutions

c composition of participant groups with different expertise levels.

\subsection{Notions and conventions}

The purpose of the present studies is to measure engineering performance depending on different experimental conditions. Within this context, it is critical to distinguish between 'experimental design' and 'aircraft design' and their related notions. The former sets conditions based on which the latter is evaluated:

a the experimental design is concerned with providing an appropriate initial setting and different conditions which are designed to tap into the process of aircraft design by observing how test persons accomplish an aircraft design task

b based on the experimental conditions, the test persons' average efficacy and efficiency of the achieved aircraft designs can be evaluated.

Furthermore, both experimental design and aircraft design may have their own inputs and outputs. To avoid confusion, we will use the notions 'variables' when referring to the experiment and 'parameters' when referring to the aircraft design. We apply the following common conventions:

c The participants generate aircraft designs by setting specific control parameters via the GUI. In this context we use input parameters and control parameters interchangeably. 
d For a set of control parameters, VAMPzero calculates certain output parameters that describe the respective aircraft design.

e Specification of control variables concern the experimental design, their variation provide different experimental conditions to be compared with one another - for instance, comparing performance for different expertise levels. In the literature control variables are sometimes also referred to as independent variables.

$\mathrm{f}$ Experimental observations are referred to as dependent variables. They are called so because the measured outcomes depend on the experimental control variables. For the aircraft design task, a dependent variable can be the best average score of an output parameter - in the experimental analysis the best average scores are then compared against a predefined experimental condition (e.g., the participants' expertise level).

\subsection{Experimental GUIs}

\subsubsection{Task simplicity}

For experimentation, GUIs are useful for several aspects. A practical advantage of the GUI is that it is a simple interface that participants can easily interact with. This opens up the opportunity to test different participant groups in an expertise level experimental condition. For instance, difference of design behaviour and design success can be tested for expert versus novice engineers versus other, i.e., experts from other disciplines. This is possible because familiarity with the specific design software or data structures is not required. Instead, parameters can be adjusted with the GUI in away that is common sense to most people with basic computer skills (which is assumed to be the case for most engineering students and experts from related disciplines). In addition, an easy access to the design software puts the focus on design skills in terms of conceptual understanding about relationships between control parameters and objectives derived from the output aircraft design values.

With the GUI, it is possible to limit the number of control parameters that participants can manipulate within predefined ranges. Confining the design and result spaces not only keeps the task simple but also allows for direct comparison of the collected data (e.g., optimal solutions).

\subsubsection{Collecting behavioural data}

Another advantage is that a GUI allows tracking of the participants' behaviour during a design session. The cursor trajectories and any GUI actions (clicks, slider movements, etc.) are recorded with a time stamp. In addition, for each trial the selected control parameters and the resulting output parameters about the aircraft design are recorded. The latter allows evaluation of engineering performance in terms of efficacy (optimal output values) and efficiency (number of iterations needed to achieve the results). The autonomous behaviour data (trajectories and actions) can be useful to investigate how participants use the information that is provided by the GUI, and that might differ according to experimental condition. 


\subsubsection{Specifying experimental conditions}

Most importantly for our research question is that experimental conditions may differ in the quality and quantity of information that the GUI provides. We are interested to find out how the control variables information quality (e.g., plots vs. tables) and information quantity (e.g., rich vs. sparse) may influence engineering performance. Combinations of these control variables yields a list of four experimental conditions, here: plots-sparse, plots-rich, tables-sparse, and tables-rich. Each condition is realised by one respective GUI. The example of Figure 3 shows the GUI for the plots-rich condition. In the middle and bottom panels on the left, participants can see the history of their control parameters choices as well as the resulting output parameters as plots (plots condition). Details (rich condition) about the compositions of output variables, relative to which participants need to optimise the aircraft, are given in the panel on the right. For the tables conditions, the plots in the left panels are replaced by tables. And for the sparse conditions, respectively, the right-side panel with the output details is left out entirely.

\section{Empirical pilot tests}

As a next step, we conducted preliminary tests of the experiment with participants. These - so-called pilot studies - are necessary to evaluate whether the current task is a suitable design exercise. Fine-tuning aside, the pilot studies also serve to improve the GUI by surveying participants about their experiences in using it. This is particularly relevant in order to display information in a more useful way. Quality and quantity of information are the most relevant experimental variables to be tested.

\subsection{Participants}

Two mechanical engineering students were recruited toward the end of the semester from an aircraft design class. These are the advanced participants who had specific experience with aircraft design. Another two mechanical engineering students who had not yet taken courses in aircraft design were recruited as novice students. Although not specifically experienced with aircraft design, they were familiar with general requirements after taking a full semester class in air transportation systems.

In concert with the ethics regulations of the German Psychology Associations, DGP and BDP (2005), each participant signed informed consent that their participation is voluntary. Participants received thereby written and verbal instructions about the experimental procedure, the data that is collected, and how we will secure and use their data.

\subsection{Material}

Each experiment was conducted on a high-performance laptop with a $39.6 \mathrm{~cm}$ (15.6 in) display with 16:9 ratio in full HD resolution (1,920 px by 1,080 px). The experimental GUIs (Figure 3) were displayed in full-screen mode. For this two laptops were set up with the necessary software tools (Section 3.1), allowing to test up to two participants at the same time. Participants were seated such that they could not see what the other one 
was doing. They were also given blocs and pens to take notes or make sketches as needed.

\subsection{Method}

All participants were tested in the 'plots-rich' condition (cf. Section 4.2.3). The experiment began with the experimenter explaining the aircraft design task and how it can be performed with the GUI. Participants also received a one-page manual with instructions, for which they were given enough time to read through and which they could keep throughout the experiment. Next, participants filled in a short survey about their aircraft design experiences.

Before starting the experiment, a demo-version of the aircraft design GUI was demonstrated for a maximum of three trials. The experimenter assisted while participants used the GUI to complete up to three demo-trials. During these trials the experimenter explained the function of the GUI and explained what kind of information is given by the different display panels. The experimenter aimed to not give any hints that might influence the participants design decisions. Instead, these trials served purely to assure that the functionality of the GUI is clear. Data from the demo-trials is not considered in the analysis.

When the participant and experimenter agreed that the GUI functionality is clear, the experimental trials were started. In the experimental mode, participants had one hour time to complete their aircraft design optimisation. They were advised that they can commit their final results any time earlier when they considered to have found an optimal aircraft design. Participants who took longer than 50 or 55 minutes were given a note that ten, or respectively, five minutes remain until they had to commit their final designs. No limit on number of trials was set for the pilot tests. After the design sessions was over, the experimenter interviewed the participants about their experience with the task and enquired feedback on the usability of the GUI.

\subsection{Procedure}

The prerequisite software tools and the specific design task that are used in this experiment were described in Sections 3.1 and 3.2. The focus on the structure and functionality of the GUI (Figure 3), allowing participants to manipulate control parameters and to evaluate output parameters in order to interactively optimise an aircraft design. In the top left panel, the so-called control panel, participants can manipulate the control parameters and thus generate novel aircraft designs. Design range, wing span, and BPR are the three parameters that participants could adjust (cf. Table 1 in Section 3.2). The design range can be selected in discrete steps from a drop-down menu, while wing span and BPR can be set pseudo continuously with sliders. To begin a new trial, they had to change the value of at least one parameter. On each trial they had also to comment on their parameter selection. Only then the button 'run VAMPzero' was released. Pressing 'run VAMPzero' commits the parameters and starts a VAMPzero iteration. While VAMPzero is calculating, which takes about 5-7 seconds, the control panel was deactivated to prevent changes to the control parameters.

In the middle left panel, referred to as the input display panel, participants can see the histories of their selected control parameters up to the current iteration trial. This is called the input display because it shows that parameters that are passed as inputs to VAMPzero 
to calculate the associated output values. The respective output parameters are displayed in the left bottom panel, referred to as the output display panel. Some input parameters lead to unfeasible designs (e.g., wings span is too short for the aircraft to fly). For these trials, the output values were left blank (see trial 1 in Figure 3). The participants were explicitly advised that they should optimise their designs with respect to both output parameters DOC and OEM (cf. Section 3.2). Specifically, they need to find as small as possible values for these two parameters as they measure cost and weight, respectively, both of which are factors that airlines seek to keep as small as possible.

Figure 3 Screen-shot of the experimental GUI for the plots-rich condition (see online version for colours)

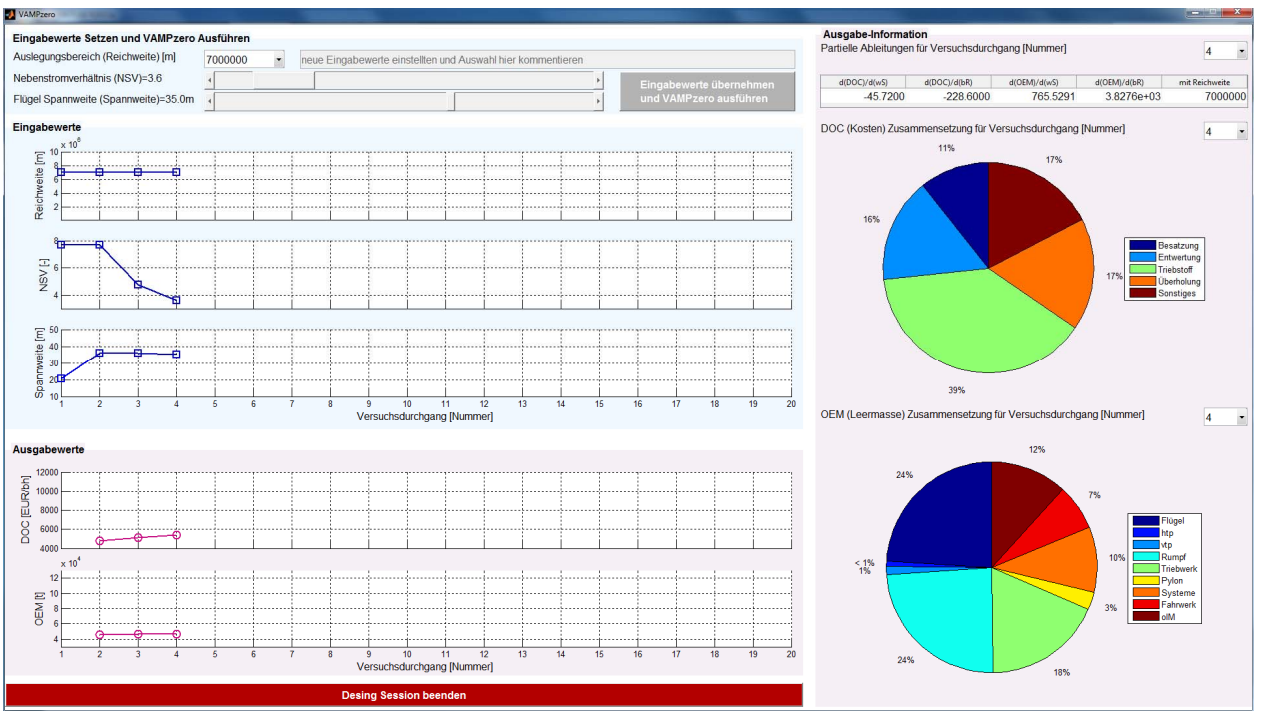

Note: The wide white borders separate the different relevant regions of interest: left-top is the control panel; left-middle is the input panel; left-bottom is the output panel; left-most-bottom is the terminate button; and right is the panel with additional information.

The left panel, so-called additional information panel, displays detailed information about the outputs for the feasible aircraft designs. It shows topmost the local sensitivities, calculated as partial derivatives of DOC and OEM with respect to the input parameters wing span and BPR for the fixed respective design range. Sensitivities were not calculated for the design range parameter since it could assume only discrete values (via a drop-down menu). However, we advised the participants that the local sensitivities numbers should be regarded cautiously since the approximation for the sensitivities was not fine enough to be sufficiently representative. This issue has been fixed for the newer versions of the experimental software.

Middle and bottom of the additional information panel, two pie-diagrams show, respectively, the breakdown of the output variables DOC and OEM in their major contributing factors. For every feasible trial the sensitivities matrix and the pie diagrams are automatically updated to show the latest respective information. Participants could display for previous trials via the adjacent drop-down menus. 
All active elements of the GUI, which the participants can manipulate are listed in Table 2. The single lines delimit the actions groups of similar purpose. The first three actions are from the category design selection - with these actions participants implement their design decisions - group one. The third group are actions with which the participants can evoke additional information about their designs. The second and fourth groups contain actions that are not really concerned with the design process per se. The buttons serve general actions to propagate or end the task. And the comments are just additional information for the experimenter. This classification is also relevant for the data collection and analysis.

Table 2 List of GUI actions that the participants can perform on the GUI from Figure 3

\begin{tabular}{llc}
\hline Action name & \multicolumn{1}{c}{ Active GUI element } & Group \\
\hline 'BPR' & Drop-down menu to select BPR & 1 \\
'Wing span' & Slider to adjust wing span & 1 \\
'Design range' & Slider to adjust wing span & 1 \\
'Comment' & Write-in field for commentaries on parameter choices & 2 \\
'Run VAMPzero' & Button to commit parameters and iterate VAMPzero & 2 \\
'Pop-up PD' & $\begin{array}{l}\text { Drop-down menu to select for which trial to show local } \\
\text { sensitivities of how DOC and OEM change in dependence } \\
\text { of small changes to BPR and wing span }\end{array}$ & 3 \\
'Pop-up DOC' & $\begin{array}{l}\text { Drop-down menu to select for which trial to show DOC } \\
\text { breakdown into major contributing components }\end{array}$ & 3 \\
'Pop-up OEM' & $\begin{array}{l}\text { Drop-down menu to select for which trial to show OEM } \\
\text { breakdown into major contributing components } \\
\text { 'Button end' }\end{array}$ & Button to finish design session \\
\hline
\end{tabular}

Notes: Short names for the actions are listed in the first column. Descriptions of these actions are given in the second column. Actions of different characteristics are grouped together, the groups are speared by single horizontal lines. Elements of the same group are given a number code, as displayed in the last column.

\subsection{Data collection}

During and after the experimental task the following data-items ware collected:

- Parameters: All input and output parameters (cf. Table 1) were recorded for each trial: design range, wing span, BPR, DOC, OEM, trial number. For trials with non-feasible designs, the outputs were set to not a number $(\mathrm{NaN})$.

- Comments: Comments that the participants enter in the control panel were recorded with a trial stamp. At least one comment per trial was required.

- GUI actions: Any actions that participants made with the active elements of the GUI were recorded with a time stamp. A list of these actions is provided in Table 2.

- Cursor trajectories: The position of the cursor on the GUI was also recorded in normalised $x$ - and $y$-coordinates with a time stamp every 0.01 of a second.

Total: $100(x ; y)$-pairs per second. 
- Interview: Participants were asked to give feedback on the GUI and the task, after they accomplished their design session. The experimenter performed the informal interviews and took written notes.

Note: Due to a path definition error in the experimental software, the parameters and cursor trajectories were not recorded for the two advanced students. This error was fixed before the novice students participated in the task.

\subsection{Analysis of pilot studies}

Note that we have tested so far only four participants, two advanced and two novice mechanical engineering students, and all with the same visualisation condition, plots-rich. The pilot tests are important in order to improve the experiment. In particular they serve to fine-tune the difficulty of the aircraft design task, but also to improve the overall experience of doing the exercise. These are the issues that we are mostly concerned with when analysing the pilot data.

\subsubsection{Efficiency and efficacy variables}

The efficacy variables are reported in Table 3. Efficacy is measured as time in minutes and number of trials needed to achieve the an optimal design. We did not analyse the efficiency (minimal scores achieved) due the small probe (collected only for the novices). Several interesting observations stick out from the table, most of them concern the efficacy variables time and number of trials. Firstly, all participants finished within less than an hour, which means that they have come to a conclusion in finding a design they consider optimal.

Table 3 Efficacy scores for the participants' aircraft designs

\begin{tabular}{lcc}
\hline \multirow{2}{*}{ Participant } & \multicolumn{2}{c}{ Efficacy: effort invested } \\
\cline { 2 - 3 } & Time [minutes] & Trials [number] \\
\hline Advanced 1 & 26 & 31 \\
Advanced 2 & 25 & 11 \\
Novice 1 & 56 & 19 \\
Novice 2 & 55 & 97 \\
\hline
\end{tabular}

Secondly, within expertise groups, both Advanced students and both Novices have similar time scores to one another. The Advanced finished in about 25-26 minutes, while the Novices in about 55-56 minutes. Overall, Novices had to work almost twice as long as the Advanced to achieve similar results. This observation hints that we can expect the task to show different levels of difficulty for the different expertise groups.

Thirdly, despite similar times within the groups, the number of iteration trials show large differences among participants in each expertise group. This is interesting as it might hint at individual differences in the strategies used: systematic exploration of the parameter space versus usage of previous knowledge for strategic setting of parameters. However, the number of participants is too low and further investigation is necessary to analyse such effects. Critical for our research is that the collection of response patterns, beyond the mere efficiency and efficacy measurements, can be revealing about the decision making process in the design task. 
Overall, a critical conclusion from these observations is that we need to limit not only the time but also the number of iterations that are allowed before the design session is over. This will help to increase the aircraft design task difficulty, which is critical in order to improve the experiment as reported in the interviews in Section 5.6.3.

\subsubsection{Behavioural data}

The evidence from the efficacy variables is also reflected by the data from the GUI actions in Figure 4 - these are the same actions, in the same order, and the same grouping as reported in the experimental procedure (Section 5.4, Table 2). Participants who solved the task more effectively, i.e., who used less trials to conclude their design, also have a lesser zig-zag pattern in their trajectories over a similar period of time as their cohorts such patterns indicate a more systematic approach, focusing on effects of single variables. In addition, analysing such patterns (measuring the density of the zig-zag) might be important when the number of trials is limited.

Figure 4 Participants' GUI events: each interaction on the active GUI elements is marked with a circle
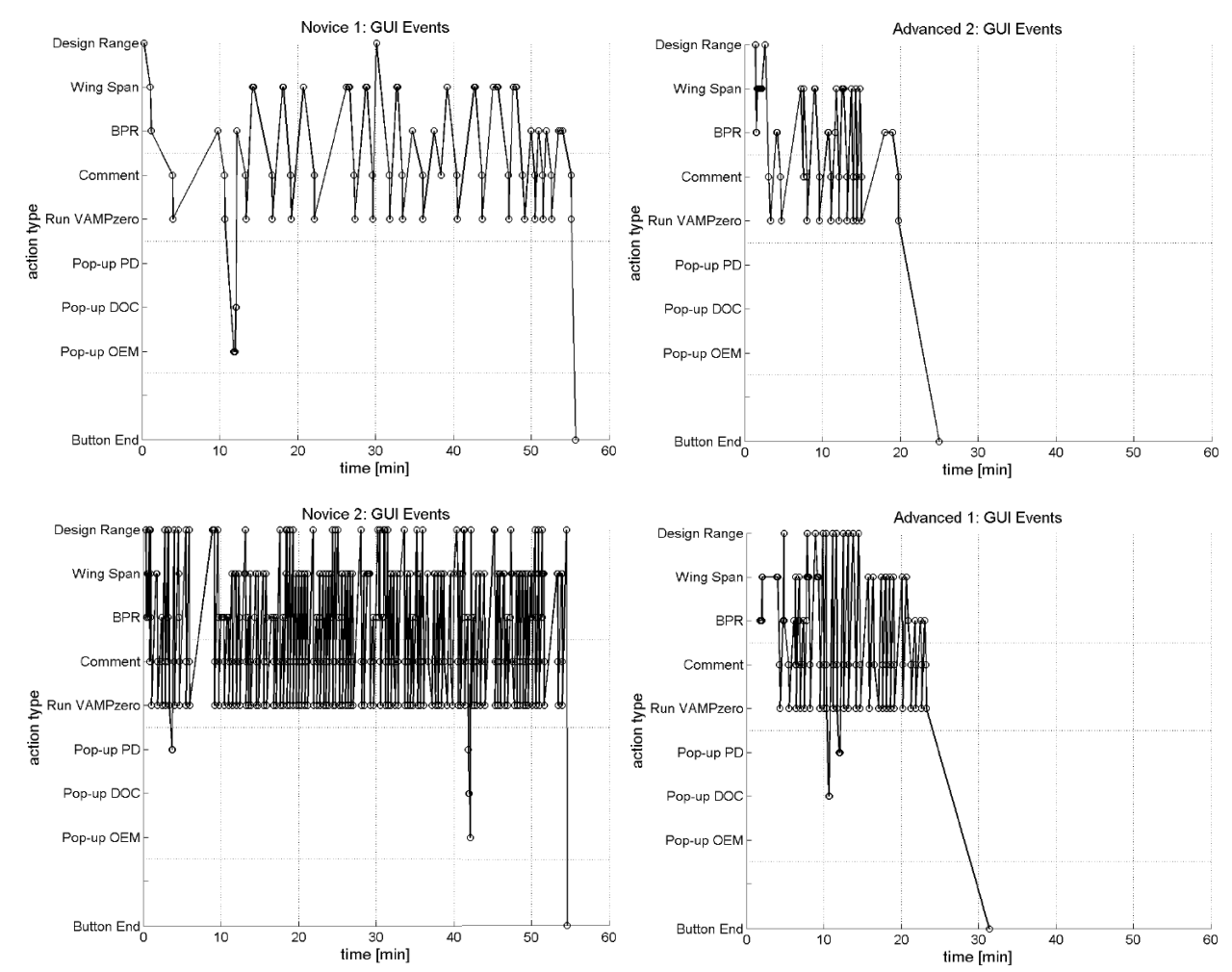

Notes: The type of the action is identified on the y-axis against time on the $\mathrm{x}$-axis. Groups of actions, as reported in Table 2, are separated by dashed horizontal lines.

The plots on the left show the novices' data, on the right are the advanced participants' data. Within the expertise groups, the plots atop are from the participants who had better scores on the efficiency and efficacy variables. 
The GUI actions, with which participants may manipulate any of the respective three input parameters, displayed atop. When comparing the plots only with respect to these three actions, another commonality between the participants, who used less trials than their cohorts, can be observed: They hardly manipulate the design range parameter, they do not often change the BPR, but mostly dial on the wing span. This suggests a systematic approach, but more data is needed to see if this really can be likened to better performance scores. For the participants who used more trials, the manipulations are more similarly distributed over the three input parameters.

In the second and the bottom action-groups are the so-called general actions entering comments, starting a trial or finishing the session - that need to be performed in order to progress through the trials or simply finish when done. They are not of much interest here, as they are per definition identical on any trial. Our focus is on the intermediate action-group, the third from the top. It shows actions, which evoke details about the output parameters from previous trials. All participants hardly ever do any actions to reveal additional information form previous trials. This clearly is a hint that this information is not used by the participants. It would also be of interest to find out why this information has not been looked at. We consider the following options:

a this information is not well selected, and simply not useful at all

b the task is too easy, such that additional information is not needed

c the additional information is relevant only for the current trial

d participants do not know how to use this information.

Further investigation is needed to reveal which option is most likely to apply. In the future experiments, we will specifically interview participants if and how they use the additional information, and to what information they would like access. Additional evidence can be revealed in the full experiment, when the rich versus sparse information conditions are compared for a larger number of participants. These two conditions directly address the question of whether the additional information we have selected can support or disturb design decisions - if group performance is better in the Rich or Sparse, respectively.

To answer such questions, ideally, one should track the participants eye movements while they perform the task (see also Hasse et al., 2012, 2013). This will be useful to provide insights about how participants access information when it is provided in different modes, for instance, in the tables versus plots conditions (cf. Section 4). As the equipment for eye tracking is not readily available, the cursor position trajectories can serve as a gross approximation in the first instance. Once the experiments are proved to show effects of visualisation, eye-tracking studies can be performed to gain additional insight. Examples for cursor trajectories are shown in Figure 5 for the novice participants. The participant novice 1 seems to investigate the output plots regularly as trials progress, as it is evident from the clustering of lines in the output panel, bottom left. In contrast, novice 2, despite denser trajectories due to the larger amount of trials which he performs, uses less actions to investigate to output plots early in the experiment - most cursor positions are clustered in the top area where parameters can set and designs iterated. 
Figure 5 Cursor trajectories (grey line) for the two novice participants
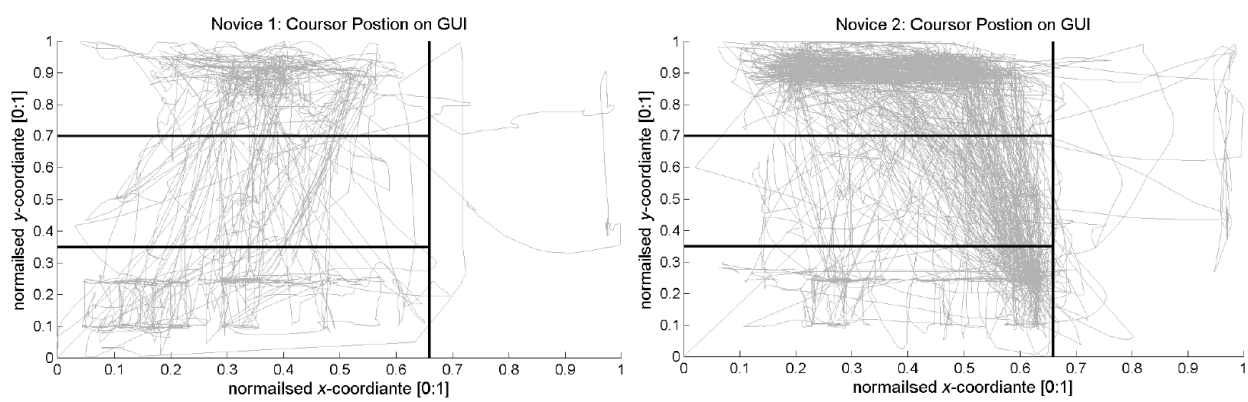

Notes: The cursor positions are encoded in normalised $(\mathrm{x} ; \mathrm{y})$-coordinates with origin in bottom left corner of the GUI. The bald black lines delimit the different regions of interest in the GUI (cf. Figure 3).

One method that we plan to pilot is to use dynamic focus on selected panels. Only the panel where the mouse cursor is hovering over will be clearly displayed, while all other panels will be blended out. This will give a hint on when and for how long participants look at the information in the different panels. However, this technique needs to be tested first as it might cause issues. For example, it would be harder for the participants to compare input and output panels as only one may be in focus.

\subsubsection{Interviews}

Given the pilot phase of the current research, the interviews were most informative on how we can improve and fine tune the experiment. The interviews, though brief were very telling about how we can improve the experiment. We will first report the feedback related to the MDO task and next the GUI related feedback.

The two advanced students participated in the first round of the experiment. From their experience with aircraft design from class, they quickly set the wing span and design range close to optimal values, which they knew by heart. They reasoned about these values also in the comments. Both of them also realised very early in the experiment that increasing BPR leads to decrease of DOC, while it had almost no effect on OEM. Note that the former is also evident from Figure 1, as the DOC surfaces monotonically increase for higher BPR values. In our numeric analysis we have overlooked that these relationships potentially trivialise the task - setting the parameter at its maximum is then always better.

A subsequent look into the VAMPzero tool and the original A320-type data file revealed that BPR, which is associated with a larger diameter does indeed not significantly increase the weight, as reported in the OEM parameter. This is because for the A320s only a limited number of engines are being produced, and the VAMPzero tools were not designed to deal with continuous changes to the BPR parameter. This issue has been now fixed.

Nonetheless, we also tested two Novice students in the version of the task that was too easy for the advanced students. They also considered the task as not very demanding or interesting. Similarly, as the advanced students reported, both novices also figured out that increasing the BPR parameter provides better results. However, they did not do so 
right at the beginning of the task, but came to this conclusion late in the session (which lasted about twice as long as for the advanced students).

From the participants, we also received important feedback that helped to improve the overall aesthetics and functionality of the GUI. One complaint was that it was very hard to adjust desired parameter values with the sliders. This was easily fixed by linking each slider with an editable text box that accepts decimals. Given the range of output parameters, differences of values may become very indistinguishable for the naked eye, and participants used the GUI option to display values when clicking on the plot. Participants find this inconvenient, as they had to try to remember or write values in order to find the minimum. We also consider this problematic, because participants are in fact generating tables from the plots - such compression actions can be identified from nearly horizontal lines in the bottom part of the cursor trajectories in Figure 5. To have a clear distinction between plots and tables condition, the option to display values is turned off for the plots. Instead a cross-hair cursor appears at the end of each trial, such that participants can use it to graphically search for minima in the output plots. Finally, elements of the third panel that belong together were grouped in functional sub-groups by using a slightly brighter colour than the background. To provide a consistent GUI appearance, the same distinction - darker background for the panel, brighter background for the elements of a group - was used in all panels.

\section{General discussion}

The numeric tests and pilot studies are preliminary steps, necessary to set-up a toolbox with which engineering performance can be measured. More specifically, the numeric tests helped narrow down a reasonable set of input and output parameters that circumscribe an MDO for an aircraft design. A GUI application was then programmed as a tool, with which engineering students can easily generate aircraft designs - trying to solve an MDO problem by adjusting input parameters in order to minimise output values (OEM and DOC for a light and cost efficient aircraft).

The pilot studies helped to identify critical improvements to both, the MDO task and the GUI. Most importantly, task difficulty had to be increased. This is realised by letting the aircraft's empty weight (OEM parameter) depend more strongly on the engines' by pass ratio (BPR parameter). In addition, time and trial limits are set, within which participants have to finish optimisation. This is to avoid that participants more or less randomly test a large as possible set of input values for an increased chance to hit optimal output values. A trials limit should encourage participants to identify meaningful relationships among parameters from the information provided by the GUI. This is absolutely critical for an extended experimental study, which aims to reveal how quality and quantity of information visualisation influences performance and behaviour.

Austin-Breneman et al. (2012) also test how students solve an MDO problem and compare the students' performance to established numeric MDO strategies. We use an MDO task to find out how people solve complex tasks, but focus on the role of visual context within which decision making takes place - these type of interactions are known from cognitive science research for fundamental forms of cognition like formation of concepts (Barsalou et al., 2007) and spatial representation (Erlhagen and Schöner, 2002; Simmering et al., 2008). By applying methods of cognitive science, we seek to 
understand how such basic interactions translate to decision making in the context of a complex engineering problem.

The main results of the present paper is a proofed and improved toolbox, with which we can study how visualisation can support engineering performance. Currently we are conducting an extended experimental series with four visualisation conditions plots-sparse, plots-rich, tables-sparse, and tables-rich - in which novice undergraduate engineering students participate. This is a critical base-line experiment that we plan to extend in several directions. For selected cases, eye-tracking will be applied for a richer insight into how participants use information. Additional measures need to be developed to link underlying performance to general innovation skills [see also Baron and Ward (2004) and Ward (2004) on cognitive science and de Boer and Badke-Schaub (2008) on physiological methods]. The experimental research needs to be supported with empirical data from interviews with engineers on how they use and would like to use visualisations. This would help to improve the GUIs by presenting information in a useful way (see also Ahmed et al., 2003; Rosenthal et al., 2013).With a good set of interactive MDO tools, the aircraft design task and GUI capabilities should be extended to study collaboration within teams of engineers [see also Austin-Breneman et al. (2012) on collaboration and Bresciani and Eppler (2009) and Eppler et al. (2013) on visualisation support of team work]. Overall, this paper presented a critical first step for the development of interactive design tools that can help enhance collaboration in multidisciplinary teams. Such tools should complement the efforts to improve the IDL as a collaborative design facility (Bachmann et al., 2012) and to provide a supportive working environment (Dineva et al., 2013). Finally, good interactive tools can also be incorporated into a project-driven educational programme to help teach collaboration early on (Dowling and Hadgraft, 2011; Mathers et al., 2012; Neerincx et al., 2006).

\section{Conclusions}

Science is just beginning to unveil processes of collaborative engineering. The current study is the foundation of a research program that is bringing empirical methods from the fields of cognitive and behavioural research into the field of engineering. We aim to contribute by developing rigorous experimental methods to study how different visualisation settings may influence engineers' decision making. Experimental research, which taps into the thought process of engineering, requires innovative techniques. Innovation, in turn, requires preliminary work as it was presented in the current paper.

The central result of this paper is to identify a design task which we believe to pose the right level of difficulty for undergraduate students. Pilot studies with Advanced and Novice participants identified critical corrections to the task and to the experimental GUI. To understand the role of visual information is central to our approach, and we anticipate the experiments to provide insights that will help to improve environments for collaborative engineering. The overarching goal is to find new ways how to enhance work experiences for individuals and outcomes for their institutions. 


\section{Acknowledgements}

This research and the Integrated Design Laboratory (IDL) are founded by the iTalent project as part of the Civil Aviation Research Programme of the federal state Hamburg, Germany. We thank Martin Spieck for initiating the iTALENT project; Daniel Böhnke and Jonas Jepsen for their assistance with the VAMPzero software-tool; and Tobias Marks for insights on how to improve the GUI.

\section{References}

Ahmed, S., Wallace, K. and Blessing, L. (2003) 'Understanding the differences between how novice and experienced designers approach design tasks', Research in Engineering Design, 21 February, Vol. 14, No. 1, pp.1-11.

Austin-Breneman, J., Honda, T. and Yang, M.C. (2012) 'A study of student design team behaviors in complex system design', Journal of Mechanical Design, Vol. 134, No. 12, p.124504, doi: $\mathrm{http}: / / \mathrm{dx}$.doi.org/10.1115/1.4007840.

Bachmann, A., Kunde, M., Litz, M., Böhnke, D. and König, S. (2010) 'Advances and work in progress in aerospace predesign data exchange, validation and software integration at the German Aerospace Center', Product Data Exchange Workshop 2010, Oslo, Norway.

Bachmann, A., Lakemeier, J. and Moerland, E. (2012) 'An integrated laboratory for collaborative design in the air transportation system', Concurrent Engineering Approaches for Sustainable Product Development in a Multi-Disciplinary Environment, September, Springer, Trier, Germany.

Baron, R.A. and Ward, T.B. (2004) 'Expanding entrepreneurial cognition's toolbox: potential contributions from the field of cognitive science', Entrepreneurship Theory and Practice, Vol. 28, No. 6, pp.553-573.

Barsalou, L.W., Breazeal, C. and Smith, L.B. (2007) 'Cognition as coordinated non-cognition', Cognitive Processing, Vol. 8, No. 2, pp.79-91, doi: 10.1007/s10339-007-0163-1.

Böhnke, D., Nagel, B. and Gollnick, V. (2011) 'An approach to multi-fidelity in conceptual aircraft design in distributed design environments', Aerospace Conference, pp.1-10.

Breazeal, C., Buchsbaum, D., Gray, J., Gatenby, D. and Blumberg, B. (2005) 'Learning from and about others: towards using imitation to bootstrap the social understanding of others by robots', in Rocha, L. and Almedia (Eds.): Artificial Life, Vol. 11, Nos. 1-2, pp.31-62, doi: $10.1162 / 1064546053278955$.

Bresciani, S. and Eppler, M.J. (2009) 'The benefits of synchronous collaborative information visualization: evidence from an experimental evaluation', Proceedings of the IEEE Information Visualisation Conference (INFOVIZ), Atlantic City, New Jersey, IEEE Press.

Buss, A.T. and Spencer, J.P. (2012) 'When seeing is knowing: the role of visual cues in the dissociation between children's rule knowledge and rule use', Journal of Experimental Child Psychology, Vol. 111, No. 3, pp.561-569.

de Boer, R. and Badke-Schaub, P. (2008) 'Emotional alignment in teams: how emotions support the design process', International Design Conference - Design, pp.1079-1086, Dubrovnik, Croatia.

DGP and BDP (2005) Ethische richtlinien der DGPs und des BDP (Deutsche Gesellschaft für Psychologie e.V. and Berufsverband Deutscher Psychologinnen und Psychologen e.V.) [online] http://www.bdp-verband.org/bdp/verband/ethik.shtml (accessed 1 December 2013).

Dineva, E., Bachmann, A., Moerland, E., Nagel, B. and Gollnick, V. (2013) 'Empirical performance evaluation in collaborative aircraft design tasks', in Bil, C., Mo, J. and Stjepandíc, J. (Eds.): 20th ISPE International Conference on Concurrent Engineering, Amsterdam, The Netherlands, pp.110-118. 
Dowling, D.G. and Hadgraft, R.G. (2011) 'A systematic consultation process to define graduate outcomes for engineering disciplines', in Hernandez, W. (Ed.): Rees 2011: Research in Engineering Education Symposium, October, Madrid, Spain, pp.525-533.

Eißfeldt, H. (2011) 'Ability requirements for air traffic controllers and pilots in future ATM systems: results from the DLR project aviator 2030', Aviation Psychology in Austria 2: Human Performance and Limitations, pp.25-37, facultas.wuv, Vienna, Austria [online] http://www.facultas.at/list?back=3497becc081a0d8ddcb4e2b38ef2882c\&xid=977313.

Eißfeldt, H., Heintz, A. and Schulze Kissing, D. (2011) 'Selection requirements to work in future ATM systems', Proceedings 16th International Symposium on Aviation Psychology, 2-5 May, Dayton, Ohio, USA, pp.315-320.

Elgh, F. (2013) 'A task oriented approach to documentation and knowledge management of systems enabling design and manufacture of highly customized products', in Bil, C., Mo, J. and Stjepandíc, J. (Eds.): 20th ISPE International Conference on Concurrent Engineering, pp.119-128, Amsterdam, The Netherlands.

Eppler, M.J. and Lengler, R. (2007) 'Towards a periodic table of visualization methods', Proceedings of the 2007 IASTED Conference on Graphics and Visualization in Engineering, Florida, USA.

Eppler, M.J., Forbes Öste, H. and Bresciani, S. (2013) 'An experimental evaluation on the impact of visual facilitation modes on idea generation in teams', International Conference Information Visualization iv13, IEEE Press, London, GB.

Erlhagen, W. and Schöner, G. (2002) 'Dynamic field theory of movement preparation', Psychological Review, Vol. 109, No. 3, pp.545-572, doi: 10.1037//0033-295X.109.3.545.

Felder, W.N. and Collopy, P. (2012) 'The elephant in the mist: what we don't know about the design, development, test and management of complex systems', Journal of Aerospace Operations, Vol. 1, No. 4, pp.317-327.

Fruchter, R., Clayton, M.J., Krawinkler, H., Kunz, J. and Teicholz, P. (1996) 'Interdisciplinary communication medium for collaborative conceptual building design', Advances in Engineering Software, March, Vol. 25, Nos. 2-3, pp.89-101.

Hasse, C., Eißfeldt, H., Maschke, P. and Bruder, C. (2013) 'Using eye tracking to describe monitoring behaviour during a passive and an active control task', in Holmqvist, K., Mulvey, F. and Johansson, R. (Eds.): Book of Abstracts of the 17th European Conference on Eye Movements, 11-16 August 2013, Vol. 6 (Journal of Eye Movement Research), Lund, Sweden.

Hasse, C., Grasshoff, D. and Bruder, C. (2012) 'How to measure monitoring performance of pilots and air traffic controllers', Proceedings of the Symposium on Eye Tracking Research and Applications, 28-30 March, Santa Barbara, CA, USA, pp.409-412 (ETRA'12 Symposium on Eye Tracking Research and Applications).

Kolonay, R.M. (2013) 'Physics-based distributed collaborative design for aerospace vehicle development and technology assessment', in Bil, C., Mo, J. and Stjepandíc, J. (Eds.): 20th ISPE International Conference on Concurrent Engineering, Amsterdam, The Netherlands, pp.198-215.

la Rocca, G.D.U.o.T. and van Tooren, M.J.L.D.U.o.T. (2008) 'Knowledge based engineering to support aircraft multidisciplinary design and optimisation', 26th International Congress of the Aeronautical Sciences, Anchorage, AL, USA, pp.1-11.

Mathers, N., Goktogan, A., Rankin, J. and Anderson, M. (2012) 'Robotic mission to mars: hands-on, minds-on, web-based learning', Acta Astronautica, Vol. 80, pp.124-131 [online] http://www.sciencedirect.com/science/journal/00945765/79.

Nagel, B., Böhnke, D., Gollnick, V., Schmollgruber, P., Rizzi, A., Rocca, G.L. and Alonso, J. (2012) 'Communication in aircraft design: can we establish a common language?', 28th International Congress of the Aeronautical Sciences, ICAS 2012, Brisbane, Australia.

Nagel, B., Zill, T., Moerland, E. and Böhnke, D. (2013) 'Virtual aircraft multidisciplinary analysis and design processes - lessons learned from the collaborative design project vamp', The 4th International Conference of the European Aerospace Societies (CEAS), Linköping, Sweden. 
Neerincx, M.A., Lindenberg, J., Smets, N., Grant, T., Bos, A., Olmedo-Soler, A. and Wolff, M. (2006) 'Cognitive engineering for long duration missions: human-machine collaboration on the Moon and Mars', Second IEEE International Conference on Space Mission Challenges for Information Technology, SMC-IT 2006, IEEE, Pasadena, CA.

Oellrich, M. and Mantwill, F. (2013) 'Focussed web based collaboration for knowledge management support', in Bil, C., Mo, J. and Stjepandíc, J. (Eds.): 20th ISPE International Conference on Concurrent Engineering, Amsterdam, The Netherlands, pp.284-292.

Patel, H., Pettitt, M. and Wilson, J.R. (2012) 'Factors of collaborative working: a framework for a collaboration model', Applied Ergonomics, Vol. 43, No. 1, pp.1-26.

Rosenthal, P., Pfeiffer, L., Müller, N.H. and Ohler, P. (2013) 'Visruption: intuitive and efficient visualization of temporal airline disruption data', in Preim, B., Rheingans, P. and Theisel, H. (Eds.): Eurographics Conference on Visualization (EUROVIS) 2013, Vol. 32.

Schubert, D., Romberg, O., Kurowski, S., Gurtuna, O., Prévot, A. and Savedra, G. (2010) 'A new knowledge management system for concurrent engineering facilities', 4th International Workshop on System \& Concurrent Engineering for Space Applications, SECESA, pp.1-9.

Simmering, V.R., Schutte, A.R. and Spencer, J.P. (2008) 'Generalizing the dynamic field theory of spatial cognition across real and developmental time scales', Brain Research, Vol. 1202, No. 4, pp.68-86 (Special Issue on Computational Cognitive Neuroscience), doi: 10.1111/j.1467-7687.2008.00700.x.

Spencer, J.P., Dineva, E. and Smith, L.B. (2009) Comment on 'Infants' perseverative search errors are induced by pragmatic misinterpretation', Science, Vol. 325, No. 5948, p.1624-a.

Ward, T.B. (2004) 'Cognition, creativity, and entrepreneurship', Journal of Business Venturing, March, Vol. 19, No. 2, pp.173-188.

$\mathrm{Xu}, \mathrm{D} ., \mathrm{Bil}, \mathrm{C}$. and Cai, G. (2013) 'Overview on the development of concurrent design facility', in Bil, C., Mo, J. and Stjepandíc, J. (Eds.): 20th ISPE International Conference on Concurrent Engineering, Amsterdam, The Netherlands, pp.550-559.

Zill, T., Ciampa, P.D. and Nagel, B. (2012) 'Multidisciplinary design optimization in a collaborative distributed aircraft design system', Aiaa-2012-0553, 50th AIAA Aerospace Sciences Meeting, Nashville, TN. 